\title{
Long-Term Prognostic Counselling in People with \\ Multiple Sclerosis Using an Online Analytical Processing Tool
}

Ricardo Kosch ${ }^{\star 1}$, Insa Schiffmann ${ }^{\star 1,2}$, Martin Daumer ${ }^{3}$, Christian Lederer $^{3}$, Antonio Scalfari ${ }^{4}$, Ian Galea ${ }^{5}$, Jutta Scheiderbauer ${ }^{6}$, Anne Rahn ${ }^{1}$ and Christoph Heesen ${ }^{1,2}$

\section{Affiliations:}

1 Institute of Neuroimmunology and Multiple Sclerosis, University Medical Center HamburgEppendorf, Martinistr. 52, D-20246 Hamburg, Germany

2 Department of Neurology, University Medical Centrer Hamburg-Eppendorf, Martinistr. 52, D-20246 Hamburg, Germany

3 Sylvia Lawry Center for Multiple Sclerosis Research \& Human Motion Institute, Munich, Germany

4 Division of Neuroinflammation and Neurodegeneration, Department of Medicine, Imperial College London, London, UK

5 Clinical Neurosciences, Clinical \& Experimental Sciences, Faculty of Medicine, University of Southampton, Southampton, UK

6 Stiftung für Selbstbestimmung und Selbstvertretung von MS-Betroffenen, Trier, Germany

* Ricardo Kosch and Insa Schiffmann are equal contributors.

\section{Contact details:}

Correspondence to:

Ricardo Kosch, Institute of Neuroimmunology and Multiple Sclerosis, University Medical Center Hamburg-Eppendorf, Martinistr. 52, D-20246 Hamburg, Germany ricardo.kosch@stud.uke.uni-hamburg.de 


\section{Abstract}

\section{Background}

Prognostic counselling is a sensitive issue in medicine and especially so in MS due to the highly heterogeneous disease course. However, people with MS (pwMS) seek prognostic information. The web-based "Evidence-Based Decision Support Tool in Multiple Sclerosis" (EBDiMS) uses data of 717 patients from the London/Ontario cohort to calculate personalized long-term prognostic information.

\section{Objective}

The aim of this study was to investigate the feasibility and effect of long-term prognostic counselling in pwMS by using EBDiMS.

\section{Methods}

Ninety consecutive pwMS were provided with personalized estimations of expected time to reach Expanded Disability Status Scale (EDSS) scores of 6 and 8 and time to conversion to secondary-progressive MS. Participants gave estimates on their own putative prognosis and rated the tool's acceptability on 6-step Likert scales.

\section{Results}

Participants rated EBDiMS as highly understandable, interesting and relevant for patientphysician encounters, coping and therapy decisions. Although it provoked a certain degree of worry in some participants, 95\% would recommend using the tool. Participants' own prognosis estimates did not change significantly following EBDiMS.

\section{Conclusion}

Long-term prognostic counselling by using an online tool has been shown to be feasible in a clinical setting. EBDiMS provides pwMS with relevant, easy-to-understand long-term prognostic information without causing relevant anxiety. 


\section{Introduction}

Multiple Sclerosis (MS) is a chronic inflammatory and degenerative disease of the central nervous system. The variability in disease progression makes estimation of prognosis challenging. While MS is benign in up to $30 \%$ of cases, it can also rapidly lead to disability. ${ }^{1-3}$ A recent UK nationwide study with 3175 people with MS (pwMS) identified a frequently unmet need for prognostic information: $76 \%$ stated to seek information on their long-term prognosis and $53 \%$ had never discussed this topic with their physician. Nearly half of the participants (48\%) even stated to have "no idea" of their long-term prognosis. ${ }^{4}$ A study conducted in Argentina in 2019 showed similar findings. ${ }^{5}$ Physicians seem to refrain from openly communicating a possible prognosis, perhaps fearing a "nocebo effect" caused by providing unfavourable information. ${ }^{6}$ This theory of "self-fulfilling prophecy" ignores the patients' right for prognostic information and to our knowledge is not built on substantial evidence. ${ }^{4,6,7}$ Although not rigorously studied in neurological patients, there is evidence from oncology that prognostic counselling might lead to increased rates of patient satisfaction and decreased rates of depression without increasing anxiety. ${ }^{6}$

Providing individualized prognosis estimates to pwMS may be a way to promote adjustment to the disease and to facilitate treatment decisions. This is especially relevant as prevalence, phenomenology and prognosis of MS seem to substantially change with an increasing prevalence of a milder disease course. ${ }^{2,3,8}$ Being confronted with an increasingly complex landscape of MS immunotherapies, a valid appraisal of one's individual prognosis together with an estimate of how disease-modifying drugs (DMDs) might alter it, should become a sine qua non for balanced shared decision-making (SDM) on treatment.

Online analytical processing tools (OLAP tools) might be a way to provide individualized prognostic information. Based on patient characteristics OLAP tools predict individual disease trajectories by using data from large longitudinal patient cohorts. In a previous study, such a tool providing short-term prognostic information was shown to be understandable and not threatening, but of moderate relevance and interest for pwMS. ${ }^{9}$ In 2013, Galea et al. reported on the "Evidence-Based Decision Support Tool in MS" (EBDiMS), which, using a matching algorithm, provides an estimation of the individualized long-term prognosis (up to 30 years) based on the data of the London/Ontario cohort gathered from 1972 to 2000. In a survey comparing prognosis estimates of 17 neurologists specialized in MS with EBDiMS' estimates, both demonstrated a good and comparable accuracy when predicting the trajectory of real-life patient cases. ${ }^{10}$

This cross-sectional study aimed to assess the feasability (i.e. understandability and acceptability), the safety and the perceived helpfulness of EBDiMS for long-term prognostic counselling for pwMS using a mixed-methods approach focusing on the patient perspective. 
We also conducted group comparisons (e.g. between different disease forms, levels of MSrelated disability and levels of anxiety/depression) on the participants' own prognostic estimates, compared them to the tool and investigated if prognostic counselling leads to changes in pwMS' risk estimates.

\section{Methods}

\section{Study design}

In this cross-sectional study with short-term follow-up that was carried out in the MS day clinic of the University Medical Centre Hamburg-Eppendorf, Germany, between October 2018 and March 2019, 90 pwMS were provided with personalized prognostic estimates of time to reach Expanded Disability Status Scale (EDSS) scores of 6 and 8, and, if applicable, conversion to secondary-progressive MS (SPMS). PWMS could participate in the study if they were 18 years and older and had clinically definite MS with EDSS $<6$. Anxiety disorders, severe depression or cognitive dysfunction (based on clinical judgement) were exclusion criteria. The project was approved by the Ethics Committee of the Hamburg Chamber of Physicians (PV6071).

After information about the study and written informed consent, eligible participants completed the pre-EBDiMS questionnaire, which asked for demographic and clinical data, and their own risk estimates regarding future disease progression. Then, they were led through the EBDiMS tool by a researcher. Prognosis estimation, referring to the historical London/Ontario cohort data as some kind of "worst case natural history" scenario, was explained in a standardized way. After using the tool, participants completed the post-EBDiMS questionnaire which asked for another estimate of their future progression risk, and their perception of the tool.

Follow-up was performed approximately 14 days afterwards via short telephone interviews (5 to 10 minutes) to re-assess the psychological acceptability (degree of worry) in the medium term and to determine if EBDiMS had a persisting and relevant impact on the participants. Therefore, the participants were asked whether (and how frequently) they thought about the study again and if they had discussed the topic with other people (e.g. their significant others, friends or relatives). We also asked the participants if they felt the need to search other sources for further information on MS long-term prognosis. 


\section{Database for Prognosis Calculations}

EBDiMS is an OLAP tool for the individualized prognosis prediction of short- and long-term prognosis in MS. It has been developed by researchers from the Sylvia Lawry Centre for MS Research (SLCMSR) using the placebo arms of randomized controlled trials, observational studies and natural history cohorts comprising $>100,000$ patient-years of data. In an external validation of the short-term tool usability and safety were confirmed..$^{9,11}$

To estimate long-term prognosis, only data from one of the best described patient cohorts, the London/Ontario cohort, was used. ${ }^{12}$ Of 717 pwMS whose data were included in the tool's algorithm, 569 pwMS had a mean follow-up of 24 years and the other 148 pwMS who passed away during the study, had a mean follow-up of 23 years. ${ }^{10}$ To estimate individual prognosis, EBDiMS identifies pwMS from the database that are similar to the study participant with respect to gender, age at first symptoms, number of relapses in the first two years and MS disease course. The tool then generates an individualized prognosis estimate of the expected time to EDSS 6, EDSS 8 and conversion to SPMS in years, which is displayed on screen. EBDiMS also displays a plot showing the average disease trajectory of the subjects chosen by the algorithm over a course of 30 years. The prognostic estimates in years refer to the median, i.e. the $50^{\text {th }}$ percentile. $^{10}$

\section{Questionnaires}

The initial versions of the two questionnaire sets (pre- and post-EBDiMS) were designed based on previous work ${ }^{9,13-15}$, relevant literature ${ }^{16}$ and the input of several researchers with different scientific backgrounds. In total, the participants answered 66 questions (41 pre-EBDiMS and 25 post-EBDiMS). The pre-EBDiMS questionnare consisted of the following parts:

(a) Hospital Anxiety and Depression Rating Scale ${ }^{17,18}$,

(b) demographic and clinical data,

(c) Patient Determined Disease Steps scale ${ }^{19}$,

(d) participants' estimates on their anticipated increase in MS-related disability over the next 5, 10 and 20 years (five-step Likert scales),

(e) participants' rating on the threat of wheelchair dependency on a visual analogue scale (range 0-10),

(f) participants' expectation if (and in how many years) they expect to reach the disability milestones EDSS 6, EDSS 8 and (for pwRRMS) conversion to SPMS (with and without DMDs).

The post-EBDiMS questionnare contained the following parts:

(g) re-assessment of the participants' risk estimates to evaluate EBDiMS' effect on these estimates: repetition of sections (d) and (f),

(h) participants' personal opinion on using the tool (e.g. if they would decide to use it again),

(i) participants' perception of the tool with respect to e.g. comprehensibility, familiarity with the information, interest, relevance, recommendation and emotional threat (sixstep Likert scales ranging from "not at all" to "absolutely"). 
The questionnaires' feasibility was tested in 5 cognitive interviews, in which the think-aloud technique was used, i.e. participants went through the questionnaires verbalizing their thoughts. ${ }^{20}$ Interviews were recorded and transcribed. To identify problems regarding wording ambiguity, comprehensibility and feasibility, analytical methods previously described by McCorry et al. (2013) were employed. A coding framework was generated from any difficulties expressed by the participants, and inconsistencies between written and verbal responses. ${ }^{21}$ Taking this framework into consideration, questionnaires were refined.

\section{Data analysis}

SPSS (version 23.0) was used for the statistical analyses; p-values of $<0.05$ were considered significant. Descriptive statistics were obtained on demographic data and perception of the prognostic information given by the tool. Paired-samples t-test or McNemar test were used to assess whether pre- and post-EBDiMS risk estimations differed significantly.

Group comparisons using non-paired t-test were employed to determine the effect of MS disease course, PDDS and HADS scores as well as disease duration on participants' estimates. To evaluate whether participants' estimates on expected progression over the next 5, 10 and 20 years (5-step Likert scales) were influenced by disease covariates or sociodemographic factors, several group comparisons using paired sample t-tests were performed. In the interest of clarity and because pre- and post-EBDiMS values of the participants' risk estimates assessed on the aforementioned Likert scales did not significantly differ, only pre-EBDiMS values will be reported in this paper.

An analysis of covariance (ANCOVA) using stepwise exclusion was performed to investigate the impact of sociodemographic factors and disease covariates on participants' estimates in years. The following covariates were included in the models: age, disease duration, PDDS, perceived progression during the last 12 months (5-step Likert scale) and HADS scores. MS disease course was included as a fixed factor.

\section{Results}

\section{Demographics}

Of 126 pwMS who were asked to participate, 36 declined. The most frequent reason for declining participation in the study was anxiety relating to the prognostic estimate. Of the final 90 pwMS (72\% relapsing-remitting MS (RRMS), 12\% secondary progressive MS (SPMS), 16\% primary progressive MS (PPMS)), 50\% were female (see table 1). Mean age was 43.8 (SD 9.5) years, mean disease duration was 9 (SD 6.9) years, and mean EDSS was 2.5 (SD 1.1). All participants stated to be interested in receiving more information on their presumed prognosis by using EBDiMS. As shown in figure 1,65\% reported they had never received 
prognostic counselling before, although $60 \%$ would have preferred it. Fifty-four percent would have liked earlier access to EBDiMS. Thirty-one percent would have wanted to use EBDiMS within the first weeks after diagnosis, and $11 \%$ stated they would have wanted to use EBDiMS, at the earliest, 10 years after diagnosis. The majority of the 45 participants receiving DMDs believed the effect of their medication on long-term prognosis to be "huge" or "very huge".

Mean score for anxiety (HADS-A) was 5.8 out of 21 (SD 3.5), mean score for depression (HADS-D) was 4.8 out of 21 (SD 4.0) (table 1). Participants were asked to rate wheelchair dependency on a scale from "not at all frightening" to "worst thing which could happen", and they reached an average score of 7.1 out of 10 points.

\section{Perception of the OLAP tool}

Overall, conveying personalized prognostic information by using EBDiMS was highly acceptable for pwMS (figure 1). On 6-step Likert scales (range 0-5, from "not at all" to "absolutely"), EBDiMS was rated as very understandable and very interesting as well as relevant for patient-physician encounters, coping with the disease and therapy decisions. Overall, EBDiMS was psychologically acceptable and did not cause significant anxiety. However, $43 \%$ of the participants claimed that EBDiMS led at least to a certain degree of worry (i.e. less than 3 out of 5 points on the Likert scale assessing psychological acceptability). Two participants who developed an acute stress reaction following EBDiMS had to be coached by a psychologist.

Despite this, 86 out of 90 (96\%) of the participants would recommend using EBDiMS, and 88 out of 90 (98\%) did not regret using the tool (figure 2).

\section{Prognostic estimates}

Following exposure to EBDiMS, the number of participants expecting to ever reach EDSS 6 if they did not take DMDs increased significantly from $78 \%$ to $88 \%(p=0.004)$ and if they did take DMDs from $52 \%$ to $71 \%$. For EDSS 8, numbers increased non-significantly from $40 \%$ to $50 \%$ without DMDs and from $24 \%$ to $29 \%$ with DMDs (table 2).

Mean prognostic estimates calculated by EBDiMS were 17.09 (SD 4.54) years to reach EDSS 6 and 25.64 (SD 5.08) years to reach EDSS 8. For pwRRMS, estimated time until SPMS conversion calculated by EBDiMS was 16.2 (SD 3.3) years. Before and after using the tool, those participants expecting to ever reach EDSS 6, EDSS 8 or to convert to SPMS estimated their future disease progression with and without DMDs, i.e. they were asked in how many years they expected to reach these disability milestones if they were or were not taking DMDs. All estimates were significantly more favourable than the ones calculated by the tool (except for time to EDSS 8 without immunotherapy pre-EBDiMS with $p=0.072$ ). Even after revealing 
the more pessimistic estimates of the tool, the participants' ones did not change significantly (table 3, table S1).

Participants also estimated how much they expected their MS-related disability to increase over the next 5, 10 and 20 years using 5-step Likert scales (ranging from "not at all" to "very severe"). These estimates, again, did not change significantly after receiving personalized prognostic information (all $\mathrm{p}>0.05$; table $\mathrm{S} 2$ ).

\section{Group comparisons}

When comparing participants with PDDS $<3$ vs. PDDS $\geq 3$, estimates concerning MS-related disability progression (5-step Likert scales, range 0-4) were significantly different between these two groups: 0.94 vs. 1.59 for 5 years; 1.31 vs. 1.87 for 10 years; 1.65 vs. 2.15 for 20 years (all $p<0.05$ ). This indicates that more severely affected participants tended to have a more negative outlook on their future disease progression (table S3). Furthermore, participants with PPMS/SPMS had a significantly more pessimistic view of their future than those with RRMS ( $p<0.05$ for 5, 10 and 20 years when comparing PPMS/SPMS vs. RRMS).

Depressed participants (HADS-D $\geq 8$ points) were more pessimistic than participants with HADS-D $<8$ points when estimating their future disease progression (all $p<0.05$ when comparing HADS-D $\geq 8$ vs. HADS-D $<8$ ). In this study population, there was also no significant difference between anxious (HADS-A $\geq 8$ points) and non-anxious participants (HADS-A <8). Disease duration did not significantly influence participants' estimates either (table S3). When comparing anxious (HADS-A $\geq 8$ ) vs. non-anxious (HADS-A <8) participants, psychological acceptability was significantly lower in anxious participants (2.26 vs $2.93 ; p=0.03$ ) (table $S 5$ ).

\section{Analysis of covariance}

After stepwise exclusion of non-significant variables, the final ANCOVAs consisted of 2 to 4 variables. Across all models, between $25.0 \%$ and $62.4 \%$ of the variance could be explained. The variables with the greatest predictive value were age, PDDS, disease duration and perceived progression during the last 12 months. $F$ - and $p$-values as well as $\eta_{p}{ }^{2}$ and $R^{2}$ are reported in table S4.

\section{Follow-up}

Follow-up was performed via telephone approximately 14 days after exposure to EBDiMS. The majority (69\%) of the 64 participants, that could be reached, stated they had thought about the study occasionally to frequently. Ninety-one percent had discussed the study with other people: $61 \%$ of them with significant others, $44 \%$ with relatives, $47 \%$ with friends and $25 \%$ with physicians. Only $20 \%$ stated to have searched other sources for further information on MS 
long-term prognosis. Degree of worry (mean 3.12 (SD 1.16); 5-step Likert scale) was not significantly different from immediately after using EBDiMS ( $p=0.398)$.

\section{Discussion}

Discussion of prognosis in medicine is a sensitive issue - especially so in MS due to the heterogeneous disease course. This cross-sectional study investigated the use of an OLAP tool providing personalized long-term prognosis estimates to 90 pwMS in order to clarify its usefulness for participants as well as its impact on their risk perceptions.

One of the main findings was that $64 \%$ of pwMS participating in this study reported never having received prognostic counselling before, although $60 \%$ would have wanted it. This is in line with findings from other recent surveys ${ }^{4,5}$, indicating a strong desire for long-term prognostic information that could possibly have helped pwMS to adjust to the disease and make complex treatment decisions.

Importantly, this study shows that prognostic counselling using the tool is feasible in a clinical setting. Participants rated EBDiMS as very understandable, interesting as well as relevant for coping, patient-physician encounters and treatment decisions. As shown in other studies, participants stated to prefer receiving prognostic information with the guidance of a doctor. ${ }^{4}$ In general, EBDiMS was psychologically acceptable and did not raise any serious safety concerns in this group of participants without known or evident anxiety disorders. However, as EBDiMS led to a certain degree of worry in $43 \%$ of pwMS and induced acute stress reactions in two participants, this needs further study. Both of these participants had a low diseaserelated knowledge as well as coping problems, suggesting that this response could be predicted by screening and potentially prevented by improving their disease-related knowledge prior to EBDiMS tool exposure. However, as we excluded pwMS with diagnosed psychiatric illnesses, our results cannot be assumed to be generalizable to pwMS with these comorbidities.

In this study, as expected, more severely affected participants had a more negative outlook on their future disease progression. PDDS, disease duration, perceived progression during the last 12 months and age were identified as the variables with the strongest influence on participants' prognostic estimates. Interestingly, participants' prognostic estimates were more optimistic compared to those calculated by EBDiMS and did not change after using the tool. This is in line with the fact that more than half of the participants stated they did not expect EBDiMS' prognostic estimates to apply to them. Participants' estimates might be more optimistic due to taking DMDs, coping strategies or even considering that MS nowadays is different from a Canadian cohort up to 50 years ago. This finding reflects the complex interplay 
of processing group estimates with ideas about one's own prognosis. Here it has been demonstrated that perception of one's own personal prognosis is better, when compared to the prognosis of individuals from a comparable group which can be considered as a major life coping mechanism. ${ }^{22}$

OLAP tools have a long history in medicine. The first attempts at an automatic evaluation of electrocardiograms with the help of computer programs were made in the late $1950 \mathrm{~s} .{ }^{23}$ Today, OLAP tools are applied in several specialties, such as obstetrics (e.g. interpretation of cardiotocograms) ${ }^{24}$ and oncology (e.g. survival prediction) ${ }^{25}$. While these tools' accuracy and validity has frequently been studied ${ }^{26,27}$ and compared against each other ${ }^{28}$, the patients' perspective has received less attention, including the patients' perception of these tools, their own estimates or the tools' influence on these estimates.

Prognosis forecasting tools are still in their infancy in neurology. Since EBDiMS is based on one of the best-described MS natural history cohorts, the London/Ontario cohort, it employs data of untreated patients that has been gathered between 1972 and $2000 .{ }^{12}$ However, the prognosis of pwMS diagnosed today seems to be different ${ }^{29,30}$ due to several reasons, including more frequent diagnosis with a milder course based on new diagnostic criteria, the so called Will Rogers phenomenon ${ }^{31}$, ascertainment bias (loss to follow-up), better general medical management, and possibly also DMDs.

Taken together, prognostic counselling using EBDiMS has been shown here to be feasible, helpful and highly appreciated by pwMS, as well as safe in this study population without diagnosed or evident psychiatric illnesses. This encourages the use of state-of-the-art predictive tools. Further research is needed to develop tools capable of providing better personalized long-term prognostic estimates that will preferably include information on the treatment effects (and risks) of DMDs. These tools could integrate information from larger registries, MRI findings and/or other biomarkers in their matching algorithms to provide more accurate and detailed estimates on long-term prognosis, possibly leading to a more positive perception of the presented information. Such estimates are not only needed when making complex treatment decisions, but will also help pwMS accept the uncertainties of their disease and thereby give them space for the hope of a more favourable course.

\section{Declaration of Conflicting Interests}

The authors declared no potential conflicts of interest with respect to the research, authorship, and/or publication of this article. 


\section{Funding}

The authors received no financial support for the research, authorship, and/or publication of this article. 


\section{References}

1. Pittock SJ, McClelland RL, Mayr WT, et al. Clinical implications of benign multiple sclerosis: A 20-year population-based follow-up study. Ann Neurol 2004; 56: 303-306.

2. Schaefer LM, Poettgen J, Fischer A, et al. Impairment and restrictions in possibly benign multiple sclerosis. Brain Behav 2019; 9: e01259.

3. Brownlee WJ, Altmann DR, Prados F, et al. Early imaging predictors of long-term outcomes in relapse-onset multiple sclerosis. Brain 2019; 142: 2276-2287.

4. Dennison L, Brown M, Kirby S, et al. Do people with multiple sclerosis want to know their prognosis? A UK nationwide study. PLoS One 2018; 13: e0193407.

5. Carnero Contentti E, López PA, Pettinicchi JP, et al. Do people with multiple sclerosis want to discuss their long-term prognosis? A nationwide study in Argentina. Mult Scler Relat Disord 2020; 37: 101445 .

6. Holloway RG, Gramling R, Kelly AG. Estimating and communicating prognosis in advanced neurologic disease. Neurology 2013; 80: 764-772.

7. Heesen C, Scalfari A, Galea I. Prognostic information for people with MS: Impossible or inevitable? Mult Scler J. Epub ahead of print October 2018. DOI: 10.1177/1352458518807101.

8. Cree BAC, Gourraud PA, Oksenberg JR, et al. Long-term evolution of multiple sclerosis disability in the treatment era. Ann Neurol 2016; 80: 499-510.

9. Heesen C, Gaissmaier W, Nguyen F, et al. Prognostic Risk Estimates of Patients with Multiple Sclerosis and Their Physicians: Comparison to an Online Analytical Risk Counseling Tool. PLoS One 2013; 8: e59042.

10. Galea I, Lederer C, Neuhaus A, et al. A Web-based tool for personalized prediction of longterm disease course in patients with multiple sclerosis. Eur J Neurol 2013; 20: 1107-1109.

11. Daumer M, Neuhaus A, Lederer C, et al. Prognosis of the individual course of disease - Steps in developing a decision support tool for Multiple Sclerosis. BMC Med Inform Decis Mak 2007; 7: 11.

12. Scalfari A, Neuhaus A, Degenhardt A, et al. The natural history of multiple sclerosis, a geographically based study 10: Relapses and long-term disability. Brain 2010; 133: 1914 1929.

13. Heesen C, Kleiter I, Nguyen F, et al. Risk perception in natalizumab-treated multiple sclerosis patients and their neurologists. Mult Scler 2010; 16: 1507-1512.

14. Kasper J, Köpke S, Mühlhauser I, et al. Evidence-based patient information about treatment of multiple sclerosis-A phase one study on comprehension and emotional responses. Patient Educ Couns 2006; 62: 56-63.

15. Köpke S, Kern S, Ziemssen $\mathrm{T}$, et al. Evidence-based patient information programme in early multiple sclerosis: A randomised controlled trial. J Neurol Neurosurg Psychiatry 2014; 85: 411418.

16. Boeije HR, Janssens ACJ. 'It might happen or it might not': how patients with multiple sclerosis explain their perception of prognostic risk. Soc Sci Med 2004; 59: 861-868.

17. Zigmond AS, Snaith RP. The Hospital Anxiety and Depression Scale. Acta Psychiatr Scand 1983; 67: 361-370.

18. Bocéréan C, Dupret E. A validation study of the hospital anxiety and depression scale (HADS) in a large sample of French employees. BMC Psychiatry 2014; 14: 354.

19. Hohol MJ, Orav EJ, Weiner HL. Disease steps in multiple sclerosis: A simple approach to evaluate disease progression. Neurology 1995; 45: 251-255.

20. Solomon $P$. The think aloud method: A practical guide to modelling cognitive processes. London: Academic Press. Epub ahead of print 1995. DOI: 10.1016/0306-4573(95)90031-4.

21. McCorry NK, Scullion L, McMurray CM, et al. Content validity of the illness perceptions questionnaire - revised among people with type 2 diabetes: A think-aloud study. Psychol Heal 2013; 28: 675-685. 
22. Sharot T, Korn CW, Dolan RJ. How unrealistic optimism is maintained in the face of reality. Nat Neurosci 2011; 14: 1475-1479.

23. Schläpfer J, Wellens HJ. Computer-Interpreted Electrocardiograms: Benefits and Limitations. J Am Coll Cardiol 2017; 70: 1183-1192.

24. Chudáček V, Spilka J, Lhotská L, et al. Automatic evaluation of intrapartum fetal heart rate recordings: a comprehensive analysis of useful features. Physiol Meas 2011; 32: 1347-1360.

25. Simmons CPL, McMillan DC, McWilliams K, et al. Prognostic Tools in Patients With Advanced Cancer: A Systematic Review. J Pain Symptom Manage 2017; 53: 962-970.e10.

26. Thurtle DR, Jenkins V, Pharoah PD, et al. Understanding of prognosis in non-metastatic prostate cancer: a randomised comparative study of clinician estimates measured against the PREDICT prostate prognostic model. Br J Cancer 2019; 121: 715-718.

27. Lau F, Downing M, Lesperance M, et al. Using the Palliative Performance Scale to Provide Meaningful Survival Estimates. J Pain Symptom Manage 2009; 38: 134-144.

28. Baba M, Maeda I, Morita T, et al. Survival prediction for advanced cancer patients in the real world: A comparison of the Palliative Prognostic Score, Delirium-Palliative Prognostic Score, Palliative Prognostic Index and modified Prognosis in Palliative Care Study predictor model. Eur J Cancer 2015; 51: 1618-1629.

29. Tremlett $\mathrm{H}$, Zhao $\mathrm{Y}$, Rieckmann $\mathrm{P}$, et al. New perspectives in the natural history of multiple sclerosis. Neurology 2010; 74: 2004-2015.

30. Montalban X. The importance of long-term data in multiple sclerosis. J Neurol 2006; 253: vi9vi15.

31. Sormani MP, Tintorè M, Rovaris M, et al. Will Rogers phenomenon in multiple sclerosis. Ann Neurol 2008; 64: 428-433. 
Table 1: Demographic data.

This table shows the demographic data of the 90 participants. Data are percentages or mean values \pm SD in brackets.

\begin{tabular}{|c|c|}
\hline female (\%) & 50 \\
\hline RRMS/SPMS/PPMS (\%) & $72 / 12 / 16$ \\
\hline on immunotherapy (\%) & 50 \\
\hline mean age (years) & $43.8(9.5)$ \\
\hline mean disease duration (years) & $9(6.9)$ \\
\hline mean EDSS & $2.5(1.1)$ \\
\hline HADS-D normal/borderline/abnormal (\%) & $74 / 14 / 11^{*}$ \\
\hline HADS-A normal/borderline/abnormal (\%) & $79 / 11 / 10$ \\
\hline mean HADS-D subscore & $4.8(4.0)$ \\
\hline mean HADS-A subscore & $5.8(3.5)$ \\
\hline \multicolumn{2}{|l|}{ *does not add up to $100 \%$ due to rounding } \\
\hline \multicolumn{2}{|c|}{$\begin{array}{l}\text { Abbreviations: SD, standard deviation; RRMS, relapsing-remitting } \\
\text { multiple sclerosis; SPMS, secondary progressive multiple sclerosis; } \\
\text { PPMS, primary progressive multiple sclerosis; EDSS, Expanded } \\
\text { Disability Status Scale; HADS-A, Hospital Anxiety and Depression Scale } \\
\text { (Anxiety); HADS-D, Hospital Anxiety and Depression Scale (Depression) }\end{array}$} \\
\hline
\end{tabular}


Table 2: Participants' prognostic estimates (yes/no answers).

This table shows the prognostic estimates (yes/no answers) of 90 participating pwMS.

\begin{tabular}{ccccc}
\hline & Item & pre-EBDiMS & post-EBDiMS & p \\
\hline $\begin{array}{c}\text { expect to reach } \\
\text { EDSS 6 }\end{array}$ & $\begin{array}{c}\text { without } \\
\text { immunotherapy }\end{array}$ & $70(77.8 \%)$ & $79(87.8 \%)$ & 0.004 \\
with immunotherapy & $47(52.2 \%)$ & $63(71.1 \%)$ & $<0.001$ \\
\hline $\begin{array}{c}\text { expect to reach } \\
\text { EDSS 8 }\end{array}$ & $\begin{array}{c}\text { without } \\
\text { immunotherapy }\end{array}$ & $36(40.0 \%)$ & $45(60.0 \%)$ & 0.064 \\
with immunotherapy & $22(24.4 \%)$ & $26(28.9 \%)$ & 0.424
\end{tabular}

Abbreviations: pwMS, person with multiple sclerosis; EBDiMS, Evidence-Based Decision Support in Multiple Sclerosis; EDSS, Expanded Disability Status Scale 
Table 3: Participants' prognostic estimates (years).

This table shows the prognostic estimates of 90 participating pwMS in years (with and without DMDs) and the tool's estimates. Data are shown as mean values \pm SD in brackets. P-values are based on paired-samples t-test.

\begin{tabular}{|c|c|c|c|c|c|c|c|}
\hline & & $\begin{array}{c}\text { Estimation } \\
\text { by } \\
\text { participants } \\
\text { pre-EBDiMS }\end{array}$ & $\begin{array}{c}\text { Estimation } \\
\text { by } \\
\text { participants } \\
\text { post- } \\
\text { EBDiMS }\end{array}$ & $\begin{array}{c}\text { p } \\
\text { pre- vs. } \\
\text { post- } \\
\text { EBDiMS }\end{array}$ & $\begin{array}{l}\text { Estimation } \\
\text { by } \\
\text { EBDiMS }\end{array}$ & $\begin{array}{c}\mathrm{p} \\
\text { Participants } \\
\text { pre-EBDiMS } \\
\text { vs. EBDiMS }\end{array}$ & $\begin{array}{c}p \\
\text { Participants } \\
\text { post- } \\
\text { EBDiMS vs. } \\
\text { EBDiMS }\end{array}$ \\
\hline $\begin{array}{l}\text { expected } \\
\text { time to }\end{array}$ & $\begin{array}{l}\text { without } \\
\text { DMDs }\end{array}$ & 20.5 (9.67) & 21.7 (8.6) & 0.269 & \multirow{2}{*}{$\begin{array}{l}17.09 \\
(4.54)\end{array}$} & 0.003 & $<0.001$ \\
\hline $\begin{array}{l}\text { EDSS } 6 \\
\text { (years) }\end{array}$ & $\begin{array}{l}\text { with } \\
\text { DMDs }\end{array}$ & 24.9 (11.9) & $25.9(10.3)$ & 0.445 & & $<0.001$ & $<0.001$ \\
\hline $\begin{array}{l}\text { expected } \\
\text { time to }\end{array}$ & $\begin{array}{l}\text { without } \\
\text { DMDs }\end{array}$ & 29.5 (11.7) & $29.1(10.2)$ & 0.090 & \multirow{2}{*}{$\begin{array}{l}25.64 \\
(5.08)\end{array}$} & 0.072 & 0.038 \\
\hline $\begin{array}{l}\text { EDSS } 8 \\
\text { (years) }\end{array}$ & $\begin{array}{l}\text { with } \\
\text { DMDs }\end{array}$ & 34.8 (11.7) & 33.1 (9.5) & 0.411 & & $<0.001$ & $<0.001$ \\
\hline
\end{tabular}

Abbreviations: pwMS, person with multiple sclerosis; EBDiMS, Evidence-Based Decision Support in Multiple Sclerosis; EDSS, Expanded Disability Status Scale; DMD, diseasemodifying drug; SD standard deviation 
Table S1: Participants' prognostic estimates (years) - time to SPMS conversion.

This table shows the estimated time of the participating pwRRMS to SPMS conversion in years (with and without DMDs) and the tool's estimates. Data are shown as mean values \pm SD in brackets. Pvalues are based on paired-samples t-test with listwise exclusion. $n=$ number of participants that gave concrete estimates.

\begin{tabular}{|c|c|c|c|c|c|c|c|}
\hline & & $\begin{array}{c}\text { Participant } \\
\text { pre- } \\
\text { EBDiMS }\end{array}$ & $\begin{array}{c}\text { Participants } \\
\text { post- } \\
\text { EBDiMS }\end{array}$ & $\begin{array}{c}\text { p } \\
\text { pre- vs. } \\
\text { post- } \\
\text { EBDiMs }\end{array}$ & EBDiMS & $\begin{array}{c}p \\
\text { Participants } \\
\text { pre- } \\
\text { EBDiMS vs. } \\
\text { EBDiMS }\end{array}$ & $\begin{array}{l}p \\
\text { Participants } \\
\text { post-EBDiMS } \\
\text { vs. EBDiMS }\end{array}$ \\
\hline \multirow{2}{*}{$\begin{array}{l}\text { expected time } \\
\text { to SPMS } \\
\text { conversion } \\
\text { (years) }\end{array}$} & $\begin{array}{l}\text { without } \\
\text { DMDs }\end{array}$ & $\begin{array}{c}21.91 \\
(10.19) \\
(n=32)\end{array}$ & $\begin{array}{l}21.38 \\
(8.33) \\
(n=52)\end{array}$ & 0.572 & $\begin{array}{l}16.17 \\
(3.27) \\
(n=52)\end{array}$ & 0.002 & $<0.001$ \\
\hline & $\begin{array}{l}\text { with } \\
\text { DMDs }\end{array}$ & $\begin{array}{l}23.64 \\
(9.38) \\
(n=18)\end{array}$ & $\begin{array}{c}27.50(10.01) \\
\quad(n=41)\end{array}$ & 0.290 & $\begin{array}{l}16.22 \\
(3.37) \\
(n=41)\end{array}$ & 0.001 & $<0.001$ \\
\hline
\end{tabular}

Abbreviations: pwRRMS, person with relapsing-remitting multiple sclerosis; SPMS, secondary progressive multiple sclerosis; EBDiMS, Evidence-Based Decision Support in Multiple Sclerosis; EDSS, Expanded Disability Status Scale; DMD, disease-modifying drug; SD, standard deviation 
Table S2: Participants' risk estimates.

This table shows the participants' risk estimates regarding the future disease progression (5-step Likert scales). P-values are based on paired-samples t-test.

pre-EBDiMS post-EBDiMS $p$

\begin{tabular}{llll}
\hline Progression 5 years (Likert 0-4) & $1.22(0.776)$ & $1.12(0.747)$ & 0.095 \\
Progression 10 years (Likert 0-4) & $1.56(0.876)$ & $1.49(0.824)$ & 0.291 \\
Progression 20 years (Likert 0-4) & $1.87(0.914)$ & $1.87(0.889)$ & 1.000
\end{tabular}

Abbreviations: EBDiMS, Evicende-Based Decision Support in Multiple Sclerosis 
Table S3: Group comparisons of risk estimates (5-step Likert scales).

This table shows the risk estimates (5-step Likert scales) of different subgroups of participants. P-values are based on paired-samples t-test.

\begin{tabular}{|c|c|c|c|}
\hline & $\begin{array}{c}\text { PDDS }<3 \\
(n=51)\end{array}$ & $\begin{array}{c}\text { PDDS } \geq 3 \\
(n=39)\end{array}$ & $\mathbf{p}$ \\
\hline Progression 5 years (Likert 0-4) & 0.94 & 1.59 & $<0.001$ \\
\hline Progression 10 years (Likert 0-4) & 1.31 & 1.87 & 0.003 \\
\hline \multirow[t]{2}{*}{ Progression 20 years (Likert 0-4) } & 1.65 & 2.15 & 0.010 \\
\hline & $\begin{array}{l}\text { RRMS } \\
(n=65)\end{array}$ & $\begin{array}{l}\text { PPMS/SPMS } \\
(n=25)\end{array}$ & $\mathbf{p}$ \\
\hline Progression 5 years (Likert 0-4) & 1.05 & 1.68 & $<0.001$ \\
\hline Progression 10 years (Likert 0-4) & 1.37 & 2.04 & 0.001 \\
\hline \multirow[t]{2}{*}{ Progression 20 years (Likert 0-4) } & 1.71 & 2.28 & 0.009 \\
\hline & $\begin{array}{c}\text { HADS-D<8 } \\
(n=67)\end{array}$ & $\begin{array}{c}\text { HADS-D } \geq 8 \\
(n=23)\end{array}$ & $\mathbf{p}$ \\
\hline Progression 5 years (Likert $0-4$ ) & 1.10 & 1.57 & 0.013 \\
\hline Progression 10 years (Likert 0-4) & 1.45 & 1.87 & 0.051 \\
\hline \multirow[t]{2}{*}{ Progression 20 years (Likert $0-4$ ) } & 1.75 & 2.22 & 0.030 \\
\hline & $\begin{array}{c}\text { HADS-A<8 } \\
(n=71)\end{array}$ & $\begin{array}{c}\text { HADS-A } \geq 8 \\
(n=19)\end{array}$ & $\mathbf{p}$ \\
\hline Progression 5 years (Likert 0-4) & 1.17 & 1.42 & 0.245 \\
\hline Progression 10 years (Likert 0-4) & 1.52 & 1.68 & 0.481 \\
\hline \multirow[t]{2}{*}{ Progression 20 years (Likert 0-4) } & 1.82 & 2.05 & 0.325 \\
\hline & $\begin{array}{c}\text { disease duration } \\
<10 \text { years } \\
(n=55)\end{array}$ & $\begin{array}{c}\text { disease duration } \\
\geq 10 \text { years } \\
(n=35)\end{array}$ & $\mathbf{p}$ \\
\hline Progression 5 years (Likert $0-4$ ) & 1.13 & 1.37 & 0.155 \\
\hline Progression 10 years (Likert 0-4) & 1.42 & 1.77 & 0.068 \\
\hline Progression 20 years (Likert 0-4) & 1.75 & 2.06 & 0.116 \\
\hline
\end{tabular}

Abbreviations: PDDS, Patient Determined Disease Steps; RRMS, relapsing-remitting multiple sclerosis; SPMS, secondary progressive multiple sclerosis; PPMS, primary progressive multiple sclerosis; HADS-A, Hospital Anxiety and Depression Scale (Anxiety); HADS-D, Hospital Anxiety and Depression Scale (Depression) 
Table S4: Analysis of covariance (ANCOVA).

This table shows the results of the final ANCOVA after stepwise exclusion of nonsignificant variables.

\begin{tabular}{|c|c|c|c|c|c|c|}
\hline Item & \multicolumn{2}{|c|}{ significant factors } & $\mathbf{F}$ & $\mathbf{p}$ & $\eta^{2}$ & $\mathbf{R}^{2}$ \\
\hline \multirow{6}{*}{$\begin{array}{c}\text { expected } \\
\text { time to EDSS } \\
6 \text { (pre- } \\
\text { EBDiMS) }\end{array}$} & \multirow{3}{*}{$\begin{array}{l}\text { without } \\
\text { immunotherapy }\end{array}$} & PDDS & 5.355 & 0.024 & 0.075 & \multirow{3}{*}{0.416} \\
\hline & & disease duration & 33.646 & $<0.001$ & 0.060 & \\
\hline & & $\begin{array}{c}\text { progression during the last } 12 \\
\text { months }\end{array}$ & 4.241 & 0.043 & 0.338 & \\
\hline & \multirow{3}{*}{ with immunotherapy } & PDDS & 13.272 & 0.001 & 0.236 & \multirow{3}{*}{0.488} \\
\hline & & age & 10.799 & 0.002 & 0.201 & \\
\hline & & $\begin{array}{c}\text { progression during the last } 12 \\
\text { months }\end{array}$ & 23.545 & $<0.001$ & 0.354 & \\
\hline \multirow{4}{*}{$\begin{array}{c}\text { expected } \\
\text { time to EDSS } \\
8 \text { (pre- } \\
\text { EBDiMS) }\end{array}$} & \multirow{2}{*}{$\begin{array}{l}\text { without } \\
\text { immunotherapy }\end{array}$} & $\begin{array}{c}\text { progression during the last } 12 \\
\text { months }\end{array}$ & 6.604 & 0.015 & 0.167 & \multirow{2}{*}{0.281} \\
\hline & & disease duration & 5.354 & 0.027 & 0.140 & \\
\hline & \multirow{2}{*}{ with immunotherapy } & MS disease form & 4.912 & 0.020 & 0.353 & \multirow{2}{*}{0.419} \\
\hline & & $\begin{array}{c}\text { progression during the last } 12 \\
\text { months }\end{array}$ & 4.734 & 0.043 & 0.208 & \\
\hline \multirow{3}{*}{$\begin{array}{c}\text { expected } \\
\text { time to SPMS } \\
\text { conversion } \\
\text { (pre- } \\
\text { EBDiMS) }\end{array}$} & $\begin{array}{l}\text { without } \\
\text { immunotherapy }\end{array}$ & disease duration & 11.347 & 0.002 & 0.274 & 0.250 \\
\hline & \multirow{2}{*}{ with immunotherapy } & $\begin{array}{l}\text { progression during the last } 12 \\
\text { months }\end{array}$ & 6.903 & 0.019 & 0.315 & \multirow{2}{*}{0.360} \\
\hline & & disease duration & 6.346 & 0.024 & 0.297 & \\
\hline \multirow{6}{*}{$\begin{array}{c}\text { expected } \\
\text { time to EDSS } \\
6 \\
\text { (post- } \\
\text { EBDiMS) }\end{array}$} & \multirow{2}{*}{$\begin{array}{l}\text { without } \\
\text { immunotherapy }\end{array}$} & PDDS & 13.675 & $<0.001$ & 0.152 & \multirow{2}{*}{0.399} \\
\hline & & disease duration & 48.343 & $<0.001$ & 0.389 & \\
\hline & \multirow{4}{*}{ with immunotherapy } & PDDS & 4.696 & 0.034 & 0.075 & \multirow{4}{*}{0.624} \\
\hline & & disease duration & 49.457 & $<0.001$ & 0.460 & \\
\hline & & $\begin{array}{c}\text { progression during the last } 12 \\
\text { months }\end{array}$ & 10.119 & 0.002 & 0.149 & \\
\hline & & age & 31.104 & $<0.001$ & 0.349 & \\
\hline \multirow{5}{*}{$\begin{array}{c}\text { expected } \\
\text { time to EDSS } \\
8 \\
\text { (post- } \\
\text { EBDiMS) }\end{array}$} & \multirow{2}{*}{$\begin{array}{l}\text { without } \\
\text { immunotherapy }\end{array}$} & $\begin{array}{c}\text { progression during the last } 12 \\
\text { months }\end{array}$ & 4.931 & 0.032 & 0.105 & \multirow{2}{*}{0.275} \\
\hline & & disease duration & 7.791 & 0.008 & 0.156 & \\
\hline & \multirow{3}{*}{ with immunotherapy } & PDDS & 6.424 & 0.046 & 0.169 & \multirow{3}{*}{0.538} \\
\hline & & disease duration & 7.021 & 0.004 & 0.326 & \\
\hline & & age & 14.889 & $<0.001$ & 0.453 & \\
\hline \multirow{7}{*}{$\begin{array}{l}\text { expected time } \\
\text { to SPMS } \\
\text { conversion } \\
\text { (post- } \\
\text { EBDiMS) }\end{array}$} & \multirow{4}{*}{$\begin{array}{l}\text { without } \\
\text { immunotherapy }\end{array}$} & PDDS & 15.335 & $<0.001$ & 0.246 & \multirow{4}{*}{0.465} \\
\hline & & disease duration & 34.726 & $<0.001$ & 0.425 & \\
\hline & & age & 4.551 & 0.038 & 0.088 & \\
\hline & & MS disease form & 5.990 & 0.018 & 0.113 & \\
\hline & \multirow{3}{*}{ with immunotherapy } & PDDS & 5.408 & 0.026 & 0.128 & \multirow{3}{*}{0.310} \\
\hline & & disease duration & 15.200 & $<0.001$ & 0.291 & \\
\hline & & age & 4.764 & 0.035 & 0.114 & \\
\hline
\end{tabular}


Abbreviations: PDDS, Patient Determined Disease Steps; RRMS, relapsing-remitting multiple sclerosis; EBDiMS, Evidence-Based Decision Support in Multiple Sclerosis; RRMS, relapsingremitting multiple sclerosis; SPMS, secondary progressive multiple sclerosis; PPMS, primary progressive multiple sclerosis 